\title{
Multi-omics integration strategies for animal epigenetic studies - A review
}

\author{
Do-Young Kim ${ }^{1}$ and Jun-Mo Kim ${ }^{1 \text { ** }}$
}

* Corresponding Author: Jun-Mo Kim Tel: +82-10-4026-5644, Fax: +82-31-675-3108,

E-mail: junmokim@cau.ac.kr

${ }^{1}$ Department of Animal Science and

Technology, Chung-Ang University, Anseong,

Gyeonggi 17546, Korea

ORCID

Do-Young Kim

https://orcid.org/0000-0003-4682-5545

Jun-Mo Kim

https://orcid.org/0000-0002-6934-398X

Submitted Jan 27, 2021; Revised Mar 13, 2021; Accepted Apr 21, 2021

\begin{abstract}
Genome-wide studies provide considerable insights into the genetic background of animals; however, the inheritance of several heritable factors cannot be elucidated. Epigenetics explains these heritabilities, including those of genes influenced by environmental factors. Knowledge of the mechanisms underlying epigenetics enables understanding the processes of gene regulation through interactions with the environment. Recently developed next-generation sequencing (NGS) technologies help understand the interactional changes in epigenetic mechanisms. There are large sets of NGS data available; however, the integrative data analysis approaches still have limitations with regard to reliably interpreting the epigenetic changes. This review focuses on the epigenetic mechanisms and profiling methods and multi-omics integration methods that can provide comprehensive biological insights in animal genetic studies.
\end{abstract}

Keywords: Epigenetic; Methylome; Transcriptome; Multi-omics Integration Analysis; Gene Regulation

\section{INTRODUCTION}

Genome-wide studies have provided considerable insights into the genetic basis of inheritance; however, they could only partially explain the heritability of complex traits [1]. Complex traits in livestock can be attributed to genetic factors; however, the final phenotypic outcome is highly dependent on the farm environment, ecotypes, and individual genetic backgrounds. This missing heritability in complex traits can be attributed to the contribution of epigenetic variability, arising from the interactions with environmental factors. Transcription, translation, and the subsequent protein modification constitute the transfer of genetic information from an archived copy of DNA to mRNA with subsequent protein production. Every cell in an organism essentially has the same DNA sequences, but the qualitative and quantitative differences in gene expression determine the cell types and functions. Regulation of gene expression is the key to differentiation and development. Epigenetics can be defined as the inheritable changes that modify DNA or related proteins without altering the DNA sequence [2]. Epigenetic information is encoded in the gene sequence and is regulated through DNA methylation, histone modification, and RNA interference (RNAi) [3]. Various epigenetic mechanisms regulate gene expression by modulating the access of transcription factors (TFs) to the regulatory regions of the gene.

DNA methylation, histone modifications, and RNAi regulate gene expression through interactions with the genetic and environmental factors, in turn influencing the phenotype, resulting in variations in various biological mechanisms. Identifying and understanding the mechanisms of epigenetics is important in a variety of traits, such as disease and pro- 
ductivity. Epigenomics provides new insights in cell biology. The development of microarray and NGS technologies enable generating genome-wide epigenetic data from large populations for investigating the characteristics of organisms and their interactions with the environment.

Epigenetic research has the potential to unravel the mechanisms underlying gene regulation through interactions with the environment; however, it remains underutilized. The epigenetic profile is continuous, dynamic, and spatiotemporally tissue-dependent, similar to the transcriptome. The advancement of NGS technology enables generating large amounts of epigenetic data and developing data analysis approaches for identifying and interpreting epigenetic changes. This review aims to discuss the key mechanisms of epigenetic regulation and the various omics data analysis methods for the characterization of the epigenetic factors throughout the genome.

\section{EPIGENETIC MECHANISMS}

The functional unit of gene expression is the chromatin; it is made up of basic units called the nucleosome, which is a complex of DNA and histone proteins. Modifications of DNA and histone proteins alter chromatin structure and subsequently influence gene expression. It is important to understand the process of reversible changes in gene activity, which are modulated through various epigenetic mechanisms (Figure 1).

\section{Histone modification}

Histone is the core protein component of the chromatin complex; it provides a structural backbone for the DNA to wrap around at regular intervals to form the chromatin. The nucleosome represents the first level of chromatin organization. It is composed of two copies each of histones $\mathrm{H} 2 \mathrm{~A}, \mathrm{H} 2 \mathrm{~B}$, $\mathrm{H} 3$, and $\mathrm{H} 4$, assembled in an octameric core with DNA tightly wrapped around the octamer [4]. Nucleosomes are unstable and change rapidly in response to external stimuli, often leading to permanent changes and contributing to disease development and progression. Modifications such as acetylation, methylation, ubiquitylation, phosphorylation, sumoylation, ribosylation, and citrullination occur posttranslationally in the amino acids of the histone proteins;

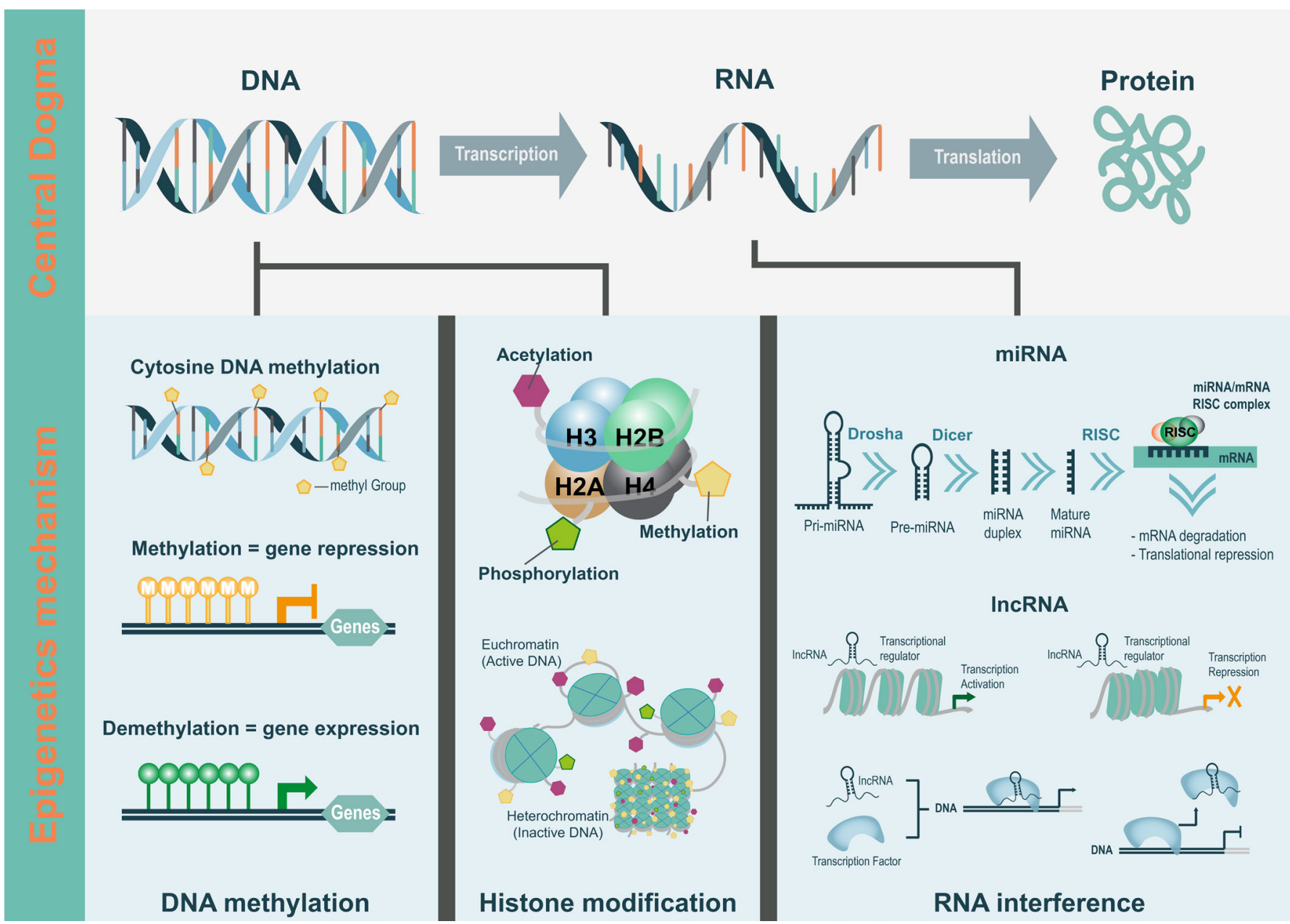

Figure 1. Genetic regulation overview by epigenetic mechanisms according to the central dogma. 
acetylation and methylation are the most extensively studied histone modifications $[5,6]$.

Histone acetylation occurs in the amino group of specific lysine residues at the $\mathrm{N}$-terminus of histone proteins [7]. Histone acetyltransferases (HATs) add acetyl groups to the tail lysine residues of histones; on the other hand, histone deacetylases remove acetyl groups from an acetylated lysine $[8,9]$. The role of histone acetylation in transcription was evaluated by identifying the causal relationship between histone acetylation and gene transcription. Several transcription cofactors have unique HATs [7,9]. HATs focus on specific gene promoters through interactions with DNAbinding regulatory factors, resulting in targeted acetylation and activation of transcription [10]. There are two mechanisms of histone acetylation, namely, charge neutralization and protein recognition/ recruitment related to transcription activation. The charge neutralization method neutralizes the positive charge in the lysine side chain, disrupting the interaction between the negatively charged DNA backbone and the lysine residue. As a result, the chromatin gets compressed, and the efficient binding of the TF to the transcription initiation site is affected [11]. The protein recognition/recruitment method causes certain histone tail acetylation patterns and other modifications in distinct sets of regulating proteins to regulate chromatin structures and functions [12-14].

Histone methylation involves transferring methyl $\left(-\mathrm{CH}_{3}\right)$ groups derived from S-adenosyl methionine to the amino acids lysine and arginine. Histone methylation is catalyzed by histone methyltransferase (HMT) and the demethylation by histone demethylase. Lysine can be mono-, di- or trimethylated; arginine can be mono-, or symmetrically or asymmetrically di-methylated [15-17]. Methylation and demethylation of histones result in the activation or inhibition of gene expression, respectively, by modulating the access of DNA to the TFs, through loosening or wrapping of the histone tail [18]. Histone methylation is predicted to be stabler than other modifications under physiological conditions; and therefore, this stability increases the possibility of histone methylation being permanent. Many histone methylations are reported in mature chromatins [11].

In contrast to histone acetylation, histone methylation does not affect the charge of histone proteins. Histone acetylation is generally correlated with transcription activation; however, histone methylation modulates transcription activation or inhibition, depending on the specific amino acid on the histone protein that is modified. Different parts of chromatin can be activated or deactivated by histone modification, depending on the methylation site [19,20]. Among the various histone methylation regions, methylation in the $\mathrm{H} 3-\mathrm{K} 4$ and H3-K9 regions is the most widely studied. Diand tri-methylation of histone $\mathrm{H} 3$ at lysine 4 (H3-K4) region is associated with transcriptional activation, similar to the acetylation of histone $\mathrm{H} 3$ at lysine 14 (H3-K14) region [21, 22]. However, di- and tri-methylation of histone $\mathrm{H} 3$ at lysine 9 (H3-K9) region results in chromatin condensation and subsequent transcription inhibition $[23,24]$. This region is the target for opposing outcomes; and therefore, the two modifications are mutually exclusive in their positioning within the chromatin.

\section{RNA interference}

The RNAi is the most recently discovered mechanism affecting epigenetic changes. Cell differentiation is modulated by regulating the expression at the gene level and chromosome level through non-coding RNAs (ncRNAs) [25-29].

The ncRNAs are not translated into proteins and are classified into housekeeping ncRNAs and regulatory ncRNAs. Regulatory RNAs are classified, based on their size, into short non-coding RNAs (sncRNAs), such as siRNAs, miRNAs, and piRNAs, and long non-coding RNAs (lncRNAs). In this study, we have reviewed miRNAs and IncRNAs, among the various ncRNAs, because they have been studied extensively for their roles in the regulation of gene expression [30,31].

miRNA is an evolutionarily conserved small single-stranded molecule (approximately 24 nucleotides). It is present in approximately $50 \%$ of the chromosomal regions prone to structural changes at the post-transcriptional level [32]. miRNAs regulate hundreds of different genes [33-36]. Unlike other small RNAs, miRNAs are derived from transcripts that form a unique hairpin structure [37]. pre-miRNAs, forming the hairpin structure, become mature miRNAs and form RNA-induced silencing complexes [38,39]. The miRNA base pairs with the mRNA through complementarity, resulting in translation inhibition or deadenylation and degradation in the $3^{\prime}$-untranslated region $[38,40]$. A study on 13,000 human genes speculated that the potential targets of miRNAs are HMTs, methyl cytosine phosphate guanine $(\mathrm{CpG})$-binding proteins, chromatin domain proteins, and histone deacetylases [34].

lncRNAs are $200 \mathrm{nt}$ or more in length and include most non-protein-coding transcripts [41]. IncRNAs are used according to the proximity to the protein-coding genes: i) sense or ii) antisense, when there is an overlap of one or more exons of another transcript on the same or opposite strand, respectively, iii) bidirectional, when the expression of the target gene and that of a neighboring coding transcript on the opposite strand are initiated in close genomic proximity, iv) intronic, when it is derived entirely from within an intron of a secondary transcript, or v) intergenic, when it lies within the genomic interval between two genes. In addition, lncRNAs have various origins, such as: i) arising from the disruption of translational reading frame of a protein-encoding gene; ii) resulting from chromosomal reorganization; for example, by 
the joining of two non-transcribed DNA regions in a manner that promotes transcription of the merged, non-coding sequences; iii) produced by replication of a non-coding gene by retrotransposition; iv) generation of a ncRNA containing adjacent repeats through partial tandem duplication; and v) arising from the insertion of transposable element(s) into a gene in a way that produces a functional, transcribed ncRNA [41]. There are no common shared mechanism in the lncRNA occurrences; however, they play similar roles in the regulation of gene expression [42]. Some lncRNAs may represent transcriptional noise or experimental artifacts; on the other hand, others serve as precursors of short RNAs; however, in many cases, they appear functional in the actual transcripts, mostly auto-regulating their own expression. Evidence that many lncRNAs are functional is confirmed through evolutionary choices regarding tissue specificity, regulation during development, localization to specific cell compartments, and association with diseases [43].

\section{DNA methylation}

DNA methylation is an epigenetic mechanism extensively studied in plants and animals. DNA methylation is the covalent modification at the $\mathrm{C}-5$ position of a cytosine residue in the DNA strand, resulting in 5-methylcytosine $(5 \mathrm{mC})[44,45]$. In mammalian somatic cells, $98 \%$ of DNA methylation occurs in a CpG sequence; on the other hand, in embryonic stem cells, only 75\% of DNA methylation occurs in CpG [44]. In addition, a significant proportion of DNA methylation is detected in non-CG sites (CHG or $\mathrm{CHH}$; where $\mathrm{H}$ can be $\mathrm{A}, \mathrm{T}$, or C), other than CpG $[46,47]$. These differentially influence the gene structure and function [48]. In mammals, transcription of most protein-coding genes is initiated at a promoter rich in CpG sequences. These CpG sequences when present in high density are known as CpG islands (CGI). There are approximately 29 million CpGs in the human genome, of which $60 \%$ to $80 \%$ are methylated $[34,46]$. Approximately $70 \%$ of annotated gene promoters are CGI-related, and CGI is mostly resistant to DNA methylation [49]. The chromatin structure adjacent to the CGI promoter facilitates transcription; on the other hand, methylated CGI causes chromatin condensation, inhibiting the onset of transcription and subsequently the gene expression. Methylation in genes is positively correlated with gene expression and may stimulate elongation and splicing [50]. In addition, DNA methylation plays a key role in normal development, genomic imprinting, X-chromosome inactivation, chromosome stability, and suppression of repetitive element transcription $[44,51]$. DNA methylation is regulated by DNA methyltransferases (DNMTs), such as DNMT1, DNMT3A, DNMT3B, and DNMT3C [50].

DNA methylation is chemically and genetically stable; however, it is a reversible modification that can occur either actively or passively. Demethylation involves the oxidation of ten-eleven translocation family enzymes (TETs) from $5 \mathrm{mC}$ to 5 -hydroxymethylcytosine $(5 \mathrm{hmC})$, which is further oxidized to 5-formylcytosine (5fC) and 5-carboxlcytosine $(5 \mathrm{caC})$ [52]. The genome-wide distribution of $5 \mathrm{hmC}$ differs from that of $5 \mathrm{mC}$. For example, in the adult human brain, $5 \mathrm{mC}$ is present in most of the gene regions; on the other hand, $5 \mathrm{hmC}$ occurs mainly in the promoter regions [53], regulating gene transcription or translation. A larger number of $5 \mathrm{hmC}$ molecules are associated with the bodies of active genes and they are often observed at the transcription start site of genes with a promoter containing a high $\mathrm{CpG}$ content. The $5 \mathrm{hmC}$ and TET proteins may modulate gene expression by regulating the chromatin accessibility of the transcriptional machinery or by inhibiting repressor binding. This is consistent with the enrichment of $5 \mathrm{hmC}$ in the gene body, promoter, and TF-binding regions [54]. DNA methylation can, therefore, serve as a distinct epigenetic marker owing to its functional role in transcriptional regulation.

\section{TRANSCRIPTOMIC AND EPIGENOMIC PROFILING}

Pyrosequencing, polymerase chain reaction (PCR), and Sanger sequencing are widely used to analyze a transcript and its methylation. These methods are precise and useful; however, they can be used only in a specific region, for identifying a small number of gene expressions. This is a limitation for evaluating a large number of samples, because of the high running time. With technological development, powerful approaches have emerged to compensate for these shortcomings.

\section{Transcriptome profiling methods}

Methods such as microarray and RNA-Seq provide a comprehensive understanding of whole-genome transcripts. These methods generate large amounts of expression data that require biological interpretation. In addition, each method has unique characteristics and requires a different analysis. Table 1 summarizes the differences between microarray and RNA-Seq techniques [55,56].

Microarray technique was developed to monitor the expression of multiple genes simultaneously. The technique originated from the large-scale mapping of genomic DNA and sequencing. Microarrays can be classified into printed, in-situ synthesized, high-density bead, or electronic, based on the characteristics of the oligo or probe, target detection, and surface support [57]. The basic principle of a microarray involves hybridization between complementary DNA strands when DNA strands (short oligos) or probes of the gene (or region to be detected) are arranged on a microchip and a fluorescently labeled target transcript is added. The transcript 
Table 1. Overview of comparison between microarray and RNA-seq approaches

\begin{tabular}{lcc}
\hline Items & Microarray [99] & RNA-seq [56] \\
\hline Principle & Hybridization & High-throughput sequencing \\
Resolution & Several to $100 \mathrm{bps}$ & Single base \\
Reference genome required & Only knowledge about the microarray & The species or closely related species \\
Different isoform & Limited & Yes \\
Discover new transcript & Limited & Yes \\
Non-coding RNA & Limited & Yes \\
\hline
\end{tabular}

abundance of a specific gene or RNA is determined based on the fluorescence intensity of each probe or short oligo, and its location on the chip provides information about the target. Microarrays generate quantitative data that yield information about the qualitative data. However, the microarray technique can only identify genes that are previously reported; it cannot predict novel or un-identified genes, and the normalization process is affected by technological variations, rather than biological differences [58].

RNA-Seq profiles the whole transcriptome, and it is the most suitable method for evaluating the expression of transcripts. Compared to the microarray methods, RNA-Seq has less background noise and wide dynamic range, and enables the detection of quantitative expression, rather than relative values. It is not limited to genomic sequences; and therefore, enables the discovery of previously unknown genes and new isoforms [59]. In addition to standard RNA sequencing methods, various types of transcriptome profiling methods exist, such as DGE-seq [60], useful for profiling specific gene expression; targeted RNA-Seq, for the detection of underexpressed genes [61]; single-cell RNA-Seq, for transcriptome studies at the single-cell level [62]; and micro RNA-Seq, for the detection of small ncRNA (less than $30 \mathrm{bp}$ ) [63]. Various platforms can be used for the analysis of RNA-Seq data, depending on the purpose and the method.

RNA-Seq involves preparing an RNA sample, synthesizing its CDNA, fragmenting the RNA, and attaching sequences essential for sequencing (such as adapters) to both ends of the fragment, for generating a library. The template strands are amplified to form clusters, and emulsion PCR methods or enzymatic amplification methods are used, depending on the platform. The sequence of the amplified template strand is analyzed using the NGS technique, and the biological significance of the generated data is evaluated for understanding the molecular mechanisms at the transcript level.

\section{DNA methylation profiling}

Changes in DNA methylation patterns are well-known mechanisms of epigenetic modification. The development of NGS technology and sequencing-based DNA methylation profiling methods enable mapping complete DNA methylomes. Three methods are available for detecting the DNA methyla- tion in the genome and generating methylation data. They include restriction enzyme-based approaches such as HpaII tiny fragments Enrichment by Ligation-mediated PCR (HELP) [64], and methylation-sensitive restriction enzyme (MRE)-seq [65]; bisulfite conversion-based approaches such as whole-genome bisulfite sequencing (WGBS) $[46,66]$, reduced-representation bisulfite sequencing (RRBS) [67], and bisulfite sequencing (BS-seq) [68]; and affinity-enrichment based approaches such as methylated DNA immunoprecipitation and sequencing (MeDIP-seq) $[65,69]$ and methylatedCpG-binding protein sequencing (MBD-seq) [70]. These provide consistent results; however, the most appropriate approach should be chosen, based on the specific biological points to be addressed. This is because the extent of genomic CpG coverage, resolution, quantitative accuracy, and cost vary widely [71-74], among the various methods. Detailed characteristics of the most commonly used genome-wide approaches are described in Table 2.

Restriction enzyme-based method uses a reagent that selectively binds to methylated DNA or cleaves DNA when it is not methylated. The MREs such as MspI, HpaII, NotI, and SmaI, form the basis of restriction enzyme-based methods.

The HELP assay restricts genomic DNA using a MRE, but employs a methylation-insensitive isoschizomer $M s p I$ as a control [75]. It provides better accuracy for both microarray and NGS-based analyses [76-78]. The control Mspl expression is affected to the same extent, regardless of methylation status, fragment size, or mutations in the locus; and therefore, expressing the HpaII signal in each gene sequence enables a better comparison among the different gene sequences in the same DNA sample [75].

In MRE-seq, only 40 to 220 bp DNA fragments can be sequencing, and the methylation status is confirmed based on the restriction of the unmethylated region. This is a timeand cost-effective sequencing method; however, it has the limitation of not reconciling the region of interest, because of the reliance on only a limited area of the genome [79].

The most common way to distinguish between methylated and unmethylated cytosine is to convert the unmethylated cytosine to uracil by treating the DNA with sodium bisulfite, while preserving the methylated cytosine [66]. Following the conversion, the uracil is converted to thymine in the PCR 
Table 2. Summary and comparison of the characteristics of global DNA methylation methods

\begin{tabular}{|c|c|c|c|}
\hline Attributes & Affinity enrichment-based & Restriction enzyme-based & Bisulfite conversion \\
\hline Assays & MeDIP-seq [81], MBD-seq [70] & HELP-seq [64], MRE-seq [65] & WGBS [46], RRBS [67] \\
\hline Resolution & Approximately 150 bp & Single base & Single base \\
\hline Advantages & $\begin{array}{l}\text { Allows for rapid and specific assessment of the average } \\
\text { methylation levels of large DNA regions, } \\
\text { No mutation introduced, Cost-effective }\end{array}$ & $\begin{array}{l}\text { High sensitivity with lower costs, } \\
\text { Simple approach, } \\
\text { Cost-effective }\end{array}$ & $\begin{array}{l}\text { Evaluates methylation status } \\
\text { of every } \mathrm{CpG} \text { site }\end{array}$ \\
\hline Limitations & $\begin{array}{l}\text { Limited by the quality and specificity } \\
\text { of the antibody or protein, } \\
\text { Bias into hyper-methylated regions, } \\
\text { Unpredictable absolute methylation level, } \\
\text { No information on separate CpG dinucleotides }\end{array}$ & $\begin{array}{l}\text { Restricted to restriction } \\
\text { enzyme-digestion sites, } \\
\text { Requires large amount, high purity, } \\
\text { and integrity of DNA }\end{array}$ & $\begin{array}{l}\text { High cost, } \\
\text { High DNA input, } \\
\text { DNA damage after bisulfite } \\
\text { conversion }\end{array}$ \\
\hline
\end{tabular}

MeDIP-seq, methylated DNA immunoprecipitation and sequencing; MBD-seq, methylated-CpG-binding protein sequencing; HELP-seq, Hpall tiny fragments Enrichment by Ligation-mediated PCR; MRE-seq , methylation-sensitive restriction enzyme; WGBS, whole-genome bisulfite sequencing; RRBS, reduced-representation bisulfite sequencing; CpGs, cytosine phosphate guanine.

step. The WGBS is the most informative and accurate method that covers the entire genome theoretically and is often used to investigate the regions outside of CGI [71,79]. It is also the most direct method with the highest resolution, for detecting methylation across the entire genome. However, it is the most expensive and a resource-demanding technique; therefore, this highly efficient method can only be employed when a comprehensive DNA methylation profile is required [80].

The RRBS is used to reduce the experimental cost of WGBS. RRBS is effective in identifying methylation of specific regions where $\mathrm{CpG}$ loci is dense, rather than that of the entire genome. The RRBS method is similar to WGBS; however, CpG-rich fragments are selected prior to bisulfite conversion and the PCR of unmethylated cytosines. Selection of fragments that are 40 to 220 bp in length covers $85 \%$ of the CGI in the promoter region [79].

Affinity-based capture methods are proposed as a costeffective alternative for sequencing only the methylated portion of the genome. In this approach, genomic DNA is fragmented and the methylated fragments are bound to either antibodies [81] or proteins with a high affinity for methylated DNA [70]. Subsequently, the unmethylated fragments are removed, and the methylation-rich portion of the genome is selected and sequenced. Depending on the affinitybased capture method used, the analytical properties may vary depending on the DNA-binding protein or antibody used.

The MeDIP-seq method employs anti-methyl cytosine antibodies. Briefly, genomic DNA is sonicated, adapters ligated to the fragments, samples denatured, and the immunoprecipitated fragments analyzed using antibodies against methylated cytosine. The immunoprecipitated DNA represent the methylated portion of the genome and is identified by comparing with the reference genome [65].

The MBD-seq is similar to MeDIP-seq, with the exception that it does not involve denaturation [73]. In addition, unlike the MeDIP method, which captures DNA fragments containing methylated cytosine, the MBD-seq method uses a protein that binds strictly to methylated CpG. MBD-seq is comprehensive with only a few exceptions, because DNA methylation in most mammalian body tissues occurs almost exclusively in CpG dinucleotides. MBD-seq is more effective in identifying methylated regions containing multiple methylated cytosines; on the other hand, MeDIP-seq is effective in identifying regions with sporadic methylated $\mathrm{CpG}$ with low biological relevance [70].

\section{MULTI-OMICS INTEGRATION ANALYSIS}

To understand the molecular complexity that underlies the various phenotypes, it is important to understand each molecule's interactions and the changes at the different molecular levels, such as at the genome, transcriptome, proteome, and metabolome levels. With the advancement of NGS technology, many biological data sets are produced at a rapid rate; however, data analysis, for providing biological insights, remains a challenge. Multi-omics integration (MOI) approach could provide comprehensive and extended biological insights. In studies using various omics data, the approach for MOI is largely focused on the statistic- and the function-based integration methods. This section describes epigenetic studies using MOI. We introduce the MOI analysis method using omics data at different molecular levels that can be applied to epigenetic research (Table 3).

\section{Epigenetics analysis using MOI strategy}

Epigenetic studies based on DNA methylation data are 
Table 3. Summary of the major multi-omics integration approaches

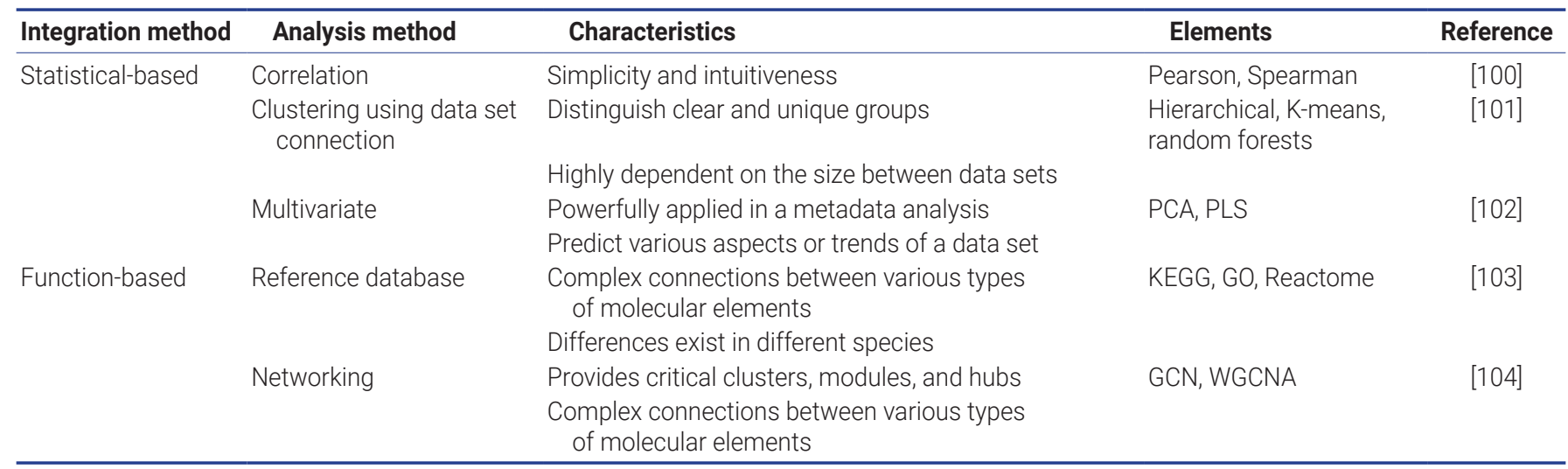

PCA, principal component analysis; PLS, partial least squares; KEGG, Kyoto encyclopedia of genes and genomes; GO, gene ontology; GCN, gene co-expression network; WGCNA, weighted gene co-expression network analysis.

widely employed in humans, livestock, and plant species. Most epigenetic studies use single data on the mechanisms of DNA methylation, histone modification, and RNA interference. Most of these studies only provide predictions of the impact on gene expression, based on the changes in epigenetic mechanisms. High-resolution and high-throughput data are generated with advances in technology; however, there is difficulty in establishing an effective approach that can facilitate combined data analysis with other omics data. Here is a summary of the recent observations of epigenetic changes in multi-omics data using a variety of data integration analyses.

Transcriptome and methylome data are generally used for evaluating epigenetic changes using the MOI method. A common method of integration is to use gene overlap of multiple omics data. There are three ways of gene overlap: i) Entire overlap between the identified differentially methylated genes (DMGs) and differentially expressed genes (DEGs), ii) overlap based on methylation and expression levels, iii) overlap between DMGs and DEGs based on gene components [82-84]. The entire overlapping method is a simple and intuitive method for finding the relationship by using the overlapping genes of the entire DMGs and DEGs. The overlapping method based on methylation and expression levels checks positive and negative associations through the overlapping genes of each level divided into DMGs (hypo-, hyper-) and DEGs (up-, down-). Overlapping based on gene component distinguishes DMGs according to each gene component, and the association between the gene component methylation and gene expression is confirmed through the overlapping. However, the accuracy is compromised based on the method of producing genetic data and the DMG profiling tool used; in addition, this technique is difficult to use when there are no overlapping genes.

In addition to the gene superposition methods, various databases and methods are used for direct (physical) and in- direct (functional) analyses [85-87]. The most common is to build a PPI network, a gene interaction network that identifies sets of genes that can interact with each other, using the STRING database $[83,88]$. Using the TF database, we can construct networks that provide the orientation and relationship to the target gene along with the motif enrichment analysis [89]. Finally, the network can be configured using statistical correlation coefficients. Constructing a network helps understand the direct relationship between the two genes and the mechanisms by which they are indirectly regulated. These networking methods form sub-clusters so that genes that are critical for the function and mechanism can be identified. In addition, for some genes, they provide a broader understanding of epigenetic changes, allowing exploration on directionality and potential regulatory relationships. The limitation is that this networking method is often configured using a reference database; and therefore, it may not be suitable for lesser-studied species.

\section{Statistic-based integration}

The statistic-based integration method is classified into three subgroups: i) correlation-based, ii) clustering-based using data set connection, and iii) multivariate analysis. The correlation-based integration approach finds the relationships between elements in one data set and those in another. The advantage of this integration is its simplicity and intuitiveness. Correlation-based integration is mainly performed using Pearson and Spearman correlation coefficients, which evaluate linear and ranking relationships; correlation analysis can also be performed using other methods, which provide standard correlation coefficients. To understand the molecular mechanisms using the correlation-based integration method, selected DEGs in the time-series data and the correlation between the transcriptomic and metabolic changes in mice, were studied [90-92].

The clustering method using data set connection is one of 
the most conceptually simple methods for combining multiple omics data sets into a single model. It allows the grouping of omics data sets with similar properties, such as expression levels, for inferring basic connections and patterns. It includes hierarchical cluster analysis, K-means cluster analysis, and random forests approach $[93,94]$. These methods can distinguish between distinct and indistinct groups; however, it has limitations when the sizes of the connected data sets differ significantly and the pattern of elements in a small data set is dominated by the pattern of elements in the larger data set.

Multivariate analysis can integrate complex omics data sets, and is more powerfully applied in experimental design and metadata analysis. The most common multivariate techniques are principal component analysis and partial least squares [95]. This approach allows users to predict various aspects or trends of a data set, including the variance or covariance associations and to investigate dynamic relationships across transcripts, proteins, and metabolites [96].

\section{Function-based integration}

When integrating various omics data, it is necessary to understand the data against the background of existing biological knowledge of how these molecules connect. Biological understanding can be improved through pathway mapping using previously identified databases $[85-87,97]$. The use of these databases to investigate changes and associations at the molecular level in response to specific environments and stimuli is well established for investigating the enriched pathways and expressed molecular mechanisms. The databases used as references for biological metabolic pathways are KEGG (https://www.genome.jp/kegg/), GO (http://geneon tology.org/), and Reactome (https://reactome.org/). These databases can be applied to MOI analysis of the key information and pathways of omics data. However, pathway annotations across different species often provide insignificant results. Based on the correlation, the strength of the relationship across expressed molecules can be evaluated through co-expression analysis [98]. This analysis suggests important clusters, modules, and hubs for biological insights related to specific pathways or regulatory molecules in a variety of biological studies. Besides co-expression network analysis, there are various biological network-based analysis for identifying organisms and cellular mechanisms. Biological networks represent complex connections across different types of molecular elements, such as genes, proteins, and metabolites. These networks help construct subnetworks that do not rely on predefined knowledge.

\section{DISCUSSION}

The MOI approach is limited by the differences in data output, variability in data structures, noise between data production platforms, and various data analysis algorithms. These biases account for inaccurate phenotypic changes. Accurate and continuous verification of experimental design, data production, databases, and data analysis tools are required for the meaningful biological interpretation of multi-omics data. Despite these complex problems and processes, data validation through various MOI approaches will increase data availability and enable integrated analysis. Many of the currently published multi-omics studies provide a rationale for what has been known or commonly observed for a long time, and some provide new insights. Applications include personalized health and nutrition, through identifying candidate genes, drug targets, and biomarkers. Detecting true causal genes, regulatory networks, and pathways will enable improving animal health, well-being, and production. Studies in a larger population will greatly increase the usefulness of predicting phenotypes, based on genetic and epigenetic variations. These approaches can reduce the repetitive work by different groups and provide a better understanding of the complex quantitative properties and underlying biology.

\section{CONCLUSION}

Genetic studies are conducted to improve productivity indicators such as meat quality, disease susceptibility, and litter size in livestock science. The phenotype is influenced by environmental factors that modulate genetic factors; therefore, improving these indicators only through genetic factors has limitations. The molecular mechanism underlying epigenetics, in response to these environmental factors is not clearly understood. Epigenetic changes in livestock influence the physiological and developmental processes, through regulating gene expression. DNA methylation, a common mechanism of epigenetics, plays an important role in phenotypic variations. This review summarizes the mechanism of genetic regulation by epigenetic variations, methods of profiling epigenetic changes, and strategies for integrating omics data to understand molecular mechanisms. A comprehensive understanding of the epigenetic changes and the identification of novel factors could be a breakthrough for better genetic improvement in livestock.

\section{CONFLICT OF INTEREST}

We certify that there is no conflict of interest with any financial organization regarding the material discussed in the manuscript.

\section{ACKNOWLEDGMENTS}

This research was supported by the Basic Science Research 
Program through the National Research Foundation of Korea (NRF) funded by the Ministry of Education (NRF-2018R1A 6A1A03025159). This research was supported by the ChungAng University Graduate Research Scholarship in 2021.

\section{REFERENCES}

1. Eichler EE, Flint J, Gibson G, et al. Missing heritability and strategies for finding the underlying causes of complex disease. Nat Rev Genet 2010;11:446-50. https://doi.org/10.1038/nrg 2809

2. Egger G, Liang G, Aparicio A, Jones PA. Epigenetics in human disease and prospects for epigenetic therapy. Nature 2004; 429:457-63. https://doi.org/10.1038/nature02625

3. Bonasio R, Tu S, Reinberg D. Molecular signals of epigenetic states. Science 2010;330:612-6. https://doi.org/10.1126/science. 1191078

4. Luger K, Mader AW, Richmond RK, Sargent DF, Richmond TJ. Crystal structure of the nucleosome core particle at 2.8 Å resolution. Nature 1997;389:251-60. https://doi.org/10. $1038 / 38444$

5. Kouzarides T. Chromatin modifications and their function. Cell 2007;128:693-705. https://doi.org/10.1016/j.cell.2007. 02.005

6. Bannister AJ, Kouzarides T. Regulation of chromatin by histone modifications. Cell Res 2011;21:381-95. https://doi.org/ 10.1038/cr.2011.22

7. Sterner DE, Berger SL. Acetylation of histones and transcription-related factors. Microbiol Mol Biol R 2000;64:43559. https://doi.org/10.1128/Mmbr.64.2.435-459.2000

8. Davie JR, Spencer VA. Control of histone modifications. J Cell Biochem 1999;75:141-8. https://doi.org/10.1002/(SICI) 1097-4644(1999)75:32+<141::AID-JCB17>3.0.CO;2-A

9. Roth SY, Denu JM, Allis CD. Histone acetyltransferases. Annu Rev Biochem 2001;70:81-120. https://doi.org/10.1146/ annurev.biochem.70.1.81

10. Kundu TK, Palhan VB, Wang ZX, An W, Cole PA, Roeder RG. Activator-dependent transcription from chromatin in vitro involving targeted histone acetylation by $\mathrm{p} 300$. Mol Cell 2000;6:551-61. https://doi.org/10.1016/S1097-2765(00)000 $54-\mathrm{X}$

11. An W. Histone acetylation and methylation: combinatorial players for transcriptional regulation. In: Kundu TK, Dasgupta D, editors. Chromatin and disease. New York, USA: SpringerVerlag; 2007. p. 351-69. https://doi.org/10.1007/1-4020-5466$1 \_16$

12.Strahl BD, Allis CD. The language of covalent histone modifications. Nature 2000;403:41-5. https://doi.org/10.1038/47412

13. Turner BM. Histone acetylation and an epigenetic code. Bioessays 2000;22:836-45. https://doi.org/10.1002/1521-1878 (200009)22:9<836::AID-BIES9>3.0.CO;2-X

14. Jenuwein T, Allis CD. Translating the histone code. Science
2001;293:1074-80. https://doi.org/10.1126/science.1063127

15.Ng SS, Yue WW, Oppermann U, Klose RJ. Dynamic protein methylation in chromatin biology. Cell Mol Life Sci 2009;66: 407. https://doi.org/10.1007/s00018-008-8303-Z

16. Bedford MT, Clarke SG. Protein arginine methylation in mammals: who, what, and why. Mol Cell 2009;33:1-13. https:// doi.org/10.1016/j.molcel.2008.12.013

17.Lan F, Shi Y. Epigenetic regulation: methylation of histone and non-histone proteins. Sci China Ser C Life Sci 2009;52: 311-22. https://doi.org/10.1007/s11427-009-0054-Z

18. Neidhart M. DNA methylation and complex human disease. San Diego, CA, USA: Elsevier/AP, Academic Press is an imrpint of Elsevier; 2016.

19. Chen SY, Sang NL. Histone deacetylase inhibitors: the epigenetic therapeutics that repress hypoxia-inducible factors. J Biomed Biotechnol 2011;2011:Article ID 197946. https://doi. org/10.1155/2011/197946

20. Crea F, Clermont PL, Mai A, Helgason CD. Histone modifications, stem cells and prostate cancer. Curr Pharm Design 2014;20:1687-97. https://doi.org/10.2174/138161281131999 90522

21.Santos-Rosa H, Schneider R, Bannister AJ, et al. Active genes are tri-methylated at K4 of histone H3. Nature 2002;419:40711. https://doi.org/10.1038/nature01080

22. Vermeulen M, Timmers HTM. Grasping trimethylation of histone H3 at lysine 4. Epigenomics-Uk 2010;2:395-406. https://doi.org/10.2217/Epi.10.11

23. Noma K, Allis CD, Grewal SIS. Transitions in distinct histone H3 methylation patterns at the heterochromatin domain boundaries. Science 2001;293:1150-5. https://doi.org/10. 1126/science. 1064150

24.Pinskaya M, Morillon A. Histone $\mathrm{H} 3$ lysine 4 di-methylation A novel mark for transcriptional fidelity? Epigenetics-Us 2009;4:302-6. https://doi.org/10.4161/epi.4.5.9369

25.Hassan MQ, Tye CE, Stein GS, Lian JB. Non-coding RNAs: Epigenetic regulators of bone development and homeostasis. Bone 2015;81:746-56. https://doi.org/10.1016/j.bone.2015. 05.026

26. Wang KC, Chang HY. Molecular mechanisms of long noncoding RNAs. Mol Cell 2011;43:904-14. https://doi.org/10. 1016/j.molcel.2011.08.018

27. Ghildiyal M, Zamore PD. Small silencing RNAs: an expanding universe. Nat Rev Genet 2009;10:94-108. https://doi.org/10. 1038/nrg2504

28. Costa FF. Non-coding RNAs, epigenetics and complexity. Gene 2008;410:9-17. https://doi.org/10.1016/j.gene.2007. 12.008

29.Peschansky VJ, Wahlestedt C. Non-coding RNAs as direct and indirect modulators of epigenetic regulation. EpigeneticsUs 2014;9:3-12. https://doi.org/10.4161/epi.27473

30. Tang X, Feng D, Li M, et al. Transcriptomic analysis of mRNAIncRNA-miRNA interactions in hepatocellular carcinoma. 
Sci Rep 2019;9:16096. https://doi.org/10.1038/s41598-01952559-x

31.Zhang W, Tang G, Zhou S, Niu Y. LncRNA-miRNA interaction prediction through sequence-derived linear neighborhood propagation method with information combination. BMC Genomics 2019;20:946. https://doi.org/10.1186/s12864019-6284-y

32. Ruvkun G. Molecular biology. Glimpses of a tiny RNA world. Science 2001;294:797-9. https://doi.org/10.1126/science. 1066315

33. Krol J, Loedige I, Filipowicz W. The widespread regulation of microRNA biogenesis, function and decay. Nat Rev Genet 2010;11:597-610. https://doi.org/10.1038/nrg2843

34.Lewis BP, Burge CB, Bartel DP. Conserved seed pairing, often flanked by adenosines, indicates that thousands of human genes are microRNA targets. Cell 2005;120:15-20. https:// doi.org/10.1016/j.cell.2004.12.035

35. Bartel DP. MicroRNAs: target recognition and regulatory functions. Cell 2009;136:215-33. https://doi.org/10.1016/j. cell.2009.01.002

36. Rajewsky N. microRNA target predictions in animals. Nat Genet 2006;38(Suppl):S8-13. https://doi.org/10.1038/ng1798

37.Lee Y, Ahn C, Han J, et al. The nuclear RNase III Drosha initiates microRNA processing. Nature 2003;425:415-9. https://doi.org/10.1038/nature01957

38. Hutvagner G, Zamore PD. A microRNA in a multiple-turnover RNAi enzyme complex. Science 2002;297:2056-60. https://doi.org/10.1126/science.1073827

39. Mourelatos Z, Dostie J, Paushkin S, et al. miRNPs: a novel class of ribonucleoproteins containing numerous microRNAs. Genes Dev 2002;16:720-8. https://doi.org/10.1101/gad.974 702

40.Wightman B, Ha I, Ruvkun G. Posttranscriptional regulation of the heterochronic gene lin- 14 by lin- 4 mediates temporal pattern formation in C. elegans. Cell 1993;75:855-62. https:// doi.org/10.1016/0092-8674(93)90530-4

41.Ponting CP, Oliver PL, Reik W. Evolution and functions of long noncoding RNAs. Cell 2009;136:629-41. https://doi. org/10.1016/j.cell.2009.02.006

42. Cao X, Yeo G, Muotri AR, Kuwabara T, Gage FH. Noncoding RNAs in the mammalian central nervous system. Annu Rev Neurosci 2006;29:77-103. https://doi.org/10.1146/annurev. neuro.29.051605.112839

43. Wilusz JE, Sunwoo H, Spector DL. Long noncoding RNAs: functional surprises from the RNA world. Genes Dev 2009; 23:1494-504. https://doi.org/10.1101/gad.1800909

44.Jin B, Li Y, Robertson KD. DNA methylation: superior or subordinate in the epigenetic hierarchy? Genes Cancer 2011; 2:607-17. https://doi.org/10.1177/1947601910393957

45. Newell-Price J, Clark AJ, King P. DNA methylation and silencing of gene expression. Trends Endocrinol Metab 2000; 11:142-8. https://doi.org/10.1016/s1043-2760(00)00248-4
46.Lister R, Pelizzola M, Dowen RH, et al. Human DNA methylomes at base resolution show widespread epigenomic differences. Nature 2009;462:315-22. https://doi.org/10.1038/ nature 08514

47.Lee J, Jang SJ, Benoit N, et al. Presence of 5-methylcytosine in $\mathrm{CpNpG}$ trinucleotides in the human genome. Genomics 2010;96:67-72. https://doi.org/10.1016/j.ygeno.2010.03.013

48. Kim KD, El Baidouri M, Jackson SA. Accessing epigenetic variation in the plant methylome. Brief Funct Genomics 2014;13:318-27. https://doi.org/10.1093/bfgp/elu003

49. Saxonov S, Berg P, Brutlag DL. A genome-wide analysis of $\mathrm{CpG}$ dinucleotides in the human genome distinguishes two distinct classes of promoters. Proc Natl Acad Sci USA 2006; 103:1412-7. https://doi.org/10.1073/pnas.0510310103

50. Jones PA. Functions of DNA methylation: islands, start sites, gene bodies and beyond. Nat Rev Genet 2012;13:484-92. https://doi.org/10.1038/nrg3230

51.Li E. Chromatin modification and epigenetic reprogramming in mammalian development. Nat Rev Genet 2002;3:662-73. https://doi.org/10.1038/nrg887

52.Sadakierska-Chudy A, Kostrzewa RM, Filip M. A comprehensive view of the epigenetic landscape part I: DNA methylation, passive and active DNA demethylation pathways and histone variants. Neurotox Res 2015;27:84-97. https://doi.org/10.1007/ s12640-014-9497-5

53. Jin SG, Wu X, Li AX, Pfeifer GP. Genomic mapping of 5-hydroxymethylcytosine in the human brain. Nucleic Acids Res 2011;39:5015-24. https://doi.org/10.1093/nar/gkr120

54. Nestor CE, Ottaviano R, Reddington J, et al. Tissue type is a major modifier of the 5-hydroxymethylcytosine content of human genes. Genome Res 2012;22:467-77. https://doi.org/ 10.1101/gr.126417.111

55.Li X, Teng S. RNA Sequencing in Schizophrenia. Bioinform Biol Insights 2015;9:53-60. https://doi.org/10.4137/BBI.S28992

56. Wang Z, Gerstein M, Snyder M. RNA-Seq: a revolutionary tool for transcriptomics. Nat Rev Genet 2009;10:57-63. https:// doi.org/10.1038/nrg2484

57.Miller MB, Tang YW. Basic concepts of microarrays and potential applications in clinical microbiology. Clin Microbiol Rev 2009;22:611-33. https://doi.org/10.1128/CMR.00019-09

58. Russo G, Zegar C, Giordano A. Advantages and limitations of microarray technology in human cancer. Oncogene 2003; 22:6497-507. https://doi.org/10.1038/sj.onc.1206865

59. Marioni JC, Mason CE, Mane SM, Stephens M, Gilad Y. RNA-seq: an assessment of technical reproducibility and comparison with gene expression arrays. Genome Res 2008; 18:1509-17. https://doi.org/10.1101/gr.079558.108

60. Morrissy AS, Morin RD, Delaney A, et al. Next-generation tag sequencing for cancer gene expression profiling. Genome Res 2009;19:1825-35. https://doi.org/10.1101/gr.094482.109

61.Levin JZ, Berger MF, Adiconis X, et al. Targeted next-generation sequencing of a cancer transcriptome enhances detection 
of sequence variants and novel fusion transcripts. Genome Biol 2009;10:R115. https://doi.org/10.1186/gb-2009-10-10r115

62.Shapiro E, Biezuner T, Linnarsson S. Single-cell sequencingbased technologies will revolutionize whole-organism science. Nat Rev Genet 2013;14:618-30. https://doi.org/10.1038/nrg 3542

63. Hafner M, Landgraf P, Ludwig J, et al. Identification of microRNAs and other small regulatory RNAs using cDNA library sequencing. Methods 2008;44:3-12. https://doi.org/10.1016/ j.ymeth.2007.09.009

64. Kriukiene E, Labrie V, Khare T, et al. DNA unmethylome profiling by covalent capture of CpG sites. Nat Commun 2013;4:2190. https://doi.org/10.1038/ncomms3190

65. Maunakea AK, Nagarajan RP, Bilenky M, et al. Conserved role of intragenic DNA methylation in regulating alternative promoters. Nature 2010;466:253-7. https://doi.org/10.1038/ nature 09165

66. Cokus SJ, Feng S, Zhang X, et al. Shotgun bisulphite sequencing of the Arabidopsis genome reveals DNA methylation patterning. Nature 2008;452:215-9. https://doi.org/10.1038/nature 06745

67. Meissner A, Gnirke A, Bell GW, Ramsahoye B, Lander ES, Jaenisch R. Reduced representation bisulfite sequencing for comparative high-resolution DNA methylation analysis. Nucleic Acids Res 2005;33:5868-77. https://doi.org/10.1093/ nar/gki901

68. Laurent L, Wong E, Li G, et al. Dynamic changes in the human methylome during differentiation. Genome Res 2010;20:32031. https://doi.org/10.1101/gr.101907.109

69. Weber M, Davies JJ, Wittig D, et al. Chromosome-wide and promoter-specific analyses identify sites of differential DNA methylation in normal and transformed human cells. Nat Genet 2005;37:853-62. https://doi.org/10.1038/ng1598

70. Serre D, Lee BH, Ting AH. MBD-isolated Genome Sequencing provides a high-throughput and comprehensive survey of DNA methylation in the human genome. Nucleic Acids Res 2010;38:391-9. https://doi.org/10.1093/nar/gkp992

71. Yong WS, Hsu FM, Chen PY. Profiling genome-wide DNA methylation. Epigenetics Chromatin 2016;9:26. https://doi. org/10.1186/s13072-016-0075-3

72. Nair SS, Coolen MW, Stirzaker C, et al. Comparison of methylDNA immunoprecipitation (MeDIP) and methyl-CpG binding domain $(\mathrm{MBD})$ protein capture for genome-wide DNA methylation analysis reveal CpG sequence coverage bias. Epigenetics-Us 2011;6:34-44. https://doi.org/10.4161/ epi.6.1.13313

73. Harris RA, Wang T, Coarfa C, et al. Comparison of sequencingbased methods to profile DNA methylation and identification of monoallelic epigenetic modifications. Nat Biotechnol 2010; 28:1097-1105. https://doi.org/10.1038/nbt.1682

74. Bock C, Tomazou EM, Brinkman AB, et al. Quantitative comparison of genome-wide DNA methylation mapping technologies. Nat Biotechnol 2010;28:1106-14. https://doi. org/10.1038/nbt.1681

75. Greally JM. The HELP-based DNA methylation assays. In: Tost J, editor. DNA methylation protocols. Methods in Molecular Biology, vol 1708. New York, NY, USA: Humana Press; 2018. pp. 191-207. https://doi.org/10.1007/978-1-4939-7481-8 -11

76. Khulan B, Thompson RF, Ye K, et al. Comparative isoschizomer profiling of cytosine methylation: The HELP assay. Genome Res 2006;16:1046-55. https://doi.org/10.1101/gr.5273806

77.Suzuki M, Greally JM. DNA methylation profiling using HpaII tiny fragment enrichment by ligation-mediated PCR (HELP). Methods 2010;52:218-22. https://doi.org/10.1016/j. ymeth.2010.04.013

78.Suzuki M, Jing QA, Lia D, Pascual M, McLellan A, Greally JM. Optimized design and data analysis of tag-based cytosine methylation assays. Genome Biol 2010;11:R36. https://doi. org/10.1186/gb-2010-11-4-r36

79. Gu H, Smith ZD, Bock C, Boyle P, Gnirke A, Meissner A. Preparation of reduced representation bisulfite sequencing libraries for genome-scale DNA methylation profiling. Nat Protoc 2011;6:468-81. https://doi.org/10.1038/nprot.2010. 190

80. Rakyan VK, Down TA, Balding DJ, Beck S. Epigenomewide association studies for common human diseases. Nat Rev Genet 2011;12:529-41. https://doi.org/10.1038/nrg3000

81.Mohn F, Weber M, Schubeler D, Roloff TC. Methylated DNA immunoprecipitation (MeDIP). In: Tost J, editor. DNA methylation. Methods in Molecular Biology, vol 507. New York, NY, USA: Humana Press; 2009. pp. 55-64. https://doi.org/10. 1007/978-1-59745-522-0_5

82.Hu Q, Ao Q, Tan Y, Gan X, Luo Y, Zhu J. Genome-wide DNA methylation and RNA analysis reveal potential mechanism of resistance to Streptococcus agalactiae in GIFT strain of nile tilapia (Oreochromis niloticus). J Immunol 2020;204: 3182-90. https://doi.org/10.4049/jimmunol.1901496

83.Guo K, Eid SA, Elzinga SE, Pacut C, Feldman EL, Hur J. Genome-wide profiling of DNA methylation and gene expression identifies candidate genes for human diabetic neuropathy. Clin Epigenetics 2020;12:123. https://doi.org/10.1186/ s13148-020-00913-6

84.Zhang M, Yan FB, Li F, et al. Genome-wide DNA methylation profiles reveal novel candidate genes associated with meat quality at different age stages in hens. Sci Rep 2017;7:45564. https://doi.org/10.1038/srep45564

85. Kanehisa M, Goto S. KEGG: Kyoto encyclopedia of genes and genomes. Nucleic Acids Res 2000;28:27-30. https://doi. org/10.1093/nar/28.1.27

86. Franceschini A, Szklarczyk D, Frankild S, et al. STRING v9.1: protein-protein interaction networks, with increased coverage and integration. Nucleic Acids Res 2013;41:D808-15. https:// 
doi.org/10.1093/nar/gks1094

87. Fabregat A, Sidiropoulos K, Viteri G, et al. Reactome pathway analysis: a high-performance in-memory approach. BMC Bioinformatics 2017;18:142. https://doi.org/10.1186/s12859017-1559-2

88. Shen J, Zhu B. Integrated analysis of the gene expression profile and DNA methylation profile of obese patients with type 2 diabetes. Mol Med Rep 2018;17:7636-44. https://doi.org/10. 3892/mmr.2018.8804

89. Wilson S, Filipp FV. A network of epigenomic and transcriptional cooperation encompassing an epigenomic master regulator in cancer. NPJ Syst Biol Appl 2018;4:24. https:// doi.org/10.1038/s41540-018-0061-4

90. Kim JM, Park JE, Yoo I, et al. Integrated transcriptomes throughout swine oestrous cycle reveal dynamic changes in reproductive tissues interacting networks. Sci Rep 2018;8: 5436. https://doi.org/10.1038/s41598-018-23655-1

91.Lim B, Kim S, Lim KS, et al. Integrated time-serial transcriptome networks reveal common innate and tissue-specific adaptive immune responses to PRRSV infection. Vet Res 2020;51:128. https://doi.org/10.1186/s13567-020-00850-5

92.Lu Y, Boekschoten MV, Wopereis S, Muller M, Kersten S. Comparative transcriptomic and metabolomic analysis of fenofibrate and fish oil treatments in mice. Physiol Genomics 2011;43:1307-18. https://doi.org/10.1152/physiolgenomics. 00100.2011

93. Gasch AP, Eisen MB. Exploring the conditional coregulation of yeast gene expression through fuzzy k-means clustering. Genome Biol 2002;3:RESEARCH0059.1 https://doi.org/10. 1186/gb-2002-3-11-research0059

94. Acharjee A, Kloosterman B, Visser RGF, Maliepaard C. Integration of multi-omics data for prediction of phenotypic traits using random forest. BMC Bioinformatics 2016;17 (Suppl 5):180. https://doi.org/10.1186/s12859-016-1043-4

95.Meng C, Zeleznik OA, Thallinger GG, Kuster B, Gholami AM, Culhane AC. Dimension reduction techniques for the integrative analysis of multi-omics data. Brief Bioinform
2016;17:628-41. https://doi.org/10.1093/bib/bbv108

96. Meng C, Kuster B, Culhane AC, Gholami AM. A multivariate approach to the integration of multi-omics datasets. BMC Bioinformatics 2014;15:162. https://doi.org/10.1186/14712105-15-162

97. Dennis G, Jr., Sherman BT, Hosack DA, et al. DAVID: Database for annotation, visualization, and integrated discovery. Genome Biol 2003;4:R60. https://doi.org/10.1186/gb-2003-4-9-r60

98. Voigt A, Almaas E. Assessment of weighted topological overlap (wTO) to improve fidelity of gene co-expression networks. BMC Bioinformatics 2019;20:58. https://doi.org/10.1186/ s12859-019-2596-9

99.Lipshutz RJ, Fodor SP, Gingeras TR, Lockhart DJ. High density synthetic oligonucleotide arrays. Nat Genet 1999;21:20-4. https://doi.org/10.1038/4447

100. Xu W, Xu M, Wang L, et al. Integrative analysis of DNA methylation and gene expression identified cervical cancerspecific diagnostic biomarkers. Signal Transduct Target Ther 2019;4:55. https://doi.org/10.1038/s41392-019-0081-6

101. Silverbush D, Cristea S, Yanovich-Arad G, Geiger T, Beerenwinke N, Sharan R. Simultaneous integration of multi-omics data improves the identification of cancer driver modules. Cell Syst 2019;8:456-66. https://doi.org/10.1016/j.cels.2019. 04.005

102. Moreno-Estrada A, Gravel S, Zakharia F, et al. Reconstructing the population genetic history of the Caribbean. PLoS Genet 2013;9:e1003925. https://doi.org/10.1371/journal.pgen. 1003925

103. Wang L, Xiao Y, Ping Y, et al. Integrating multi-omics for uncovering the architecture of cross-talking pathways in breast cancer. PLoS One 2014;9:e104282. https://doi.org/10. 1371/journal.pone.0104282

104. Chella Krishnan K, Kurt Z, Barrere-Cain R, et al. Integration of multi-omics data from mouse diversity panel highlights mitochondrial dysfunction in non-alcoholic fatty liver disease. Cell Syst 2018;6:103-15. https://doi.org/10.1016/j.cels.2017. 12.006 